\title{
Layer-guided shear acoustic plate mode sensor
}

\author{
M. I. Newton, ${ }^{\text {a) }}$ G. McHale, and F. Martin \\ Department of Chemistry and Physics, The Nottingham Trent University, Clifton Lane, Nottingham NG11 8NS, \\ United Kingdom
}

(Received 5 December 2002; accepted 29 January 2003)

\begin{abstract}
Experimental data are presented for an acoustic wave sensor based on a layer-guided shear acoustic plate mode excited on a thin quartz substrate. The effect of coating the front and back faces with polymer waveguiding layers is shown to convert the plate modes into layer-guided plate modes in a manner analogous to Love waves and to produce a similar enhancement of mass sensitivity. These layer-guided plate mode devices offer the possibility of liquid-phase sensing with transducers situated on the face of the substrate isolated from the liquid, while offering a mass sensitivity comparable to a Love wave device. (C) 2003 American Institute of Physics.
\end{abstract}

[DOI: $10.1063 / 1.1563832]$

Acoustic wave sensors that operate in liquid media are often based on shear, horizontally polarized acoustic waves to avoid significant compressional wave generation. The Love wave configuration has one of the highest mass sensitivities, ${ }^{1}$ but for liquid-phase sensing has the disadvantage of requiring the interdigital transducers (IDTs) to be on the same face of the substrate as that exposed to the liquid. Shear horizontal acoustic plate modes (SH-APMs) may be excited and detected by IDTs situated on the opposite face of the substrate to that used for sensing, ${ }^{2,3}$ but these modes exhibit a lower mass sensitivity than Love waves. In recent theoretical work, ${ }^{4,5}$ we derived a dispersion equation for shear, horizontally polarized acoustic waves propagating in a system composed of a finite thickness substrate and a finite thickness guiding layer having a lower shear acoustic speed. The solutions of the dispersion equation were shown to describe both Love waves and layer-guided APMs. In that theoretical work, higher-order Love waves were identified as continuations from the lowest order APM associated with the previous Love wave mode and it was predicted that a layerguided APM could have a mass sensitivity comparable to that of a Love wave. In this letter, we present experimental data that directly supports these theoretical predictions.

The uncoated devices were fabricated with propagation orthogonal to the x-axis of a $250-\mu \mathrm{m}$, ST-cut quartz substrate polished on the top and bottom faces. This type of device is known to support both a ???? (SSBW) ${ }^{1}$ and shear, horizontally polarized plate modes. ${ }^{3}$ Substrates of this type, but of larger thickness, have been widely used to create Love wave biosensors. ${ }^{6}$ The IDT used a three-finger, single-double interleaved design, thus allowing operation at the fundamental frequency and at the second harmonic. Each IDT was of length $150 \lambda$ and aperture $50 \lambda$, where the wavelength $\lambda=150$ $\mu \mathrm{m}$. Finger widths and spacings were $25 \mu \mathrm{m}$ and the centerto-center distance between IDTs was $75 \lambda$. The guiding layers were deposited across the whole surface of the device and consisted of spin-coated resist (S1813 Shipley MicroElectronics), subsequently hard-baked on a hotplate at $200^{\circ} \mathrm{C}$ for $10 \mathrm{~min}$. The effects of coating each face separately and of

${ }^{a)}$ Electronic mail: michael.newton@ntu.ac.uk coating both faces were investigated. Resonant frequencies of the devices were measured at the minimum insertion loss of each mode using an Agilent 8712ET network analyzer. The uncoated SSBW resonated at $f_{\mathrm{SSBW}}=33.4 \mathrm{MHz}$; the first eight plate modes were observed to be well resolved and occurred at frequencies of 34.1, 36.4, 39.2, 43, 47.2, 51.7, 56.7, and 62.1 $\mathrm{MHz}$, respectively.

Figure 1 shows the effect of coating a polymer guiding layer onto the substrate face containing the IDTs. The $y$-axis is the frequency of the minimum insertion loss for each mode (SSBW and plate) as a function of the dimensionless parameter $z=d f / v_{l}$, where $d$ is the guiding-layer thickness, from the deposition conditions, and $v_{l}=1100 \mathrm{~ms}^{-1}$ is a constant representing the intrinsic shear acoustic speed of the polymer layer. The combination $\lambda_{l}=f / v_{l}$ is a characteristic shear acoustic wavelength for the polymer guiding layer. In Fig. 1, the plate modes have $f>f_{\text {SSBW }}$ (indicated by open triangles and open circles) and the Love wave modes have

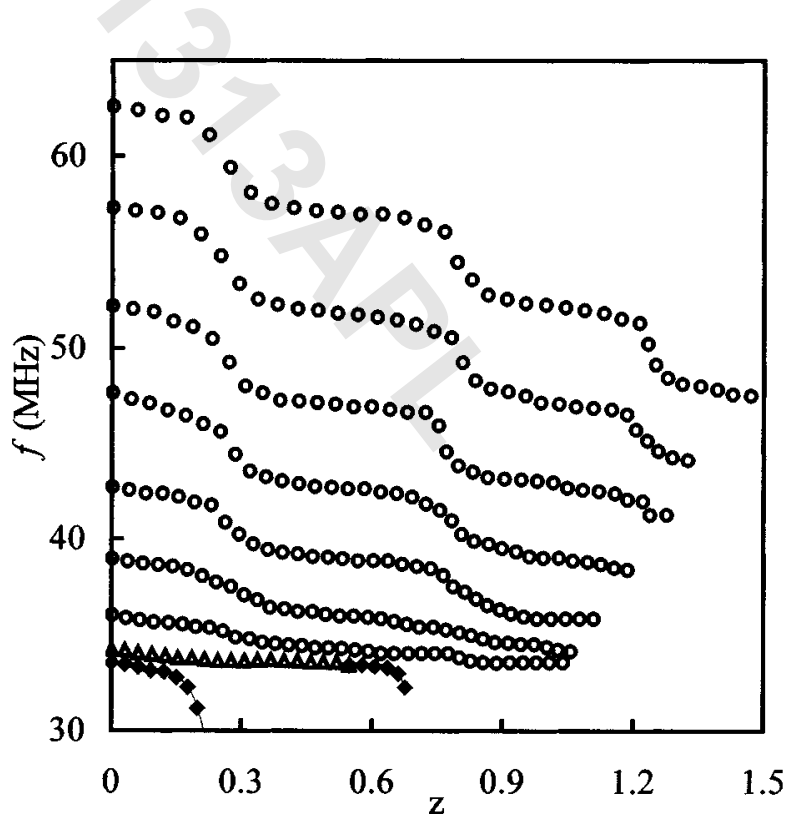

FIG. 1. Frequency of minimum insertion loss of the SSBW (solid diamonds), the lowest-order plate mode (open triangles) and the higher-order plate modes (open circles) and as a function of the normalized thickness $z$ of polymer coating on the same face as the transducers. 
$f<f_{\text {SSBW }}$ (indicated by solid diamonds). As expected, coating the polymer guiding layer onto the same face as the IDTs allows a Love wave to be created from the SSBW. The frequency of the first Love mode initially decreases slowly as the guiding layer thickness increased from zero, but then a sharp transition in frequency occurs at $z \sim 0.22$. The increase in the wave-guide-layer thickness causes the displacement of the first Love wave mode to increasingly transfer from the substrate into the guiding layer. When the layer thickness is large (i.e., $d>\lambda_{l} / 4$ ), the mode is attenuated to such an extent that it can no longer be separated from the baseline noise. This is consistent with theoretical expectations, which suggest that once the wave displacement is localized in the polymer guiding layer, the attenuation of the signal will be similar to that for a shear acoustic wave propagating in the highloss polymer. ${ }^{7}$ Theory also suggests that a higher order Love wave can occur with low-loss for thick polymer guiding layers, because its displacement will initially be supported dominantly by the substrate rather than the polymer; for thicker layers, the mode becomes attenuated as the displacement transfers into the high-loss polymer guiding layer. This pattern can be seen in Fig. 1, with the second Love wave mode occurring at $z \sim 0.55$ and subsequently fading into the noise baseline for $z>0.67$.

Consideration of the Love wave modes in Fig. 1 shows that the basic device characteristics are consistent with previous reports on Love wave sensors. However, Fig. 1 also shows that as the guiding layer thickness increases, the frequencies of the plate modes (i.e., $f>f_{\mathrm{SSBW}}$ ) reduce until each transforms into the next lower plate mode. Moreover, the second Love mode can be identified as the continuation from the lowest order layer-guided APM (shown as open triangles). The step-like cascading pattern in the plate modes with increasing guiding layer thickness is consistent with the theoretical predictions and has significant consequences for the mass sensitivity of the modes. The high mass sensitivity of a Love wave device can be understood directly from the experimental dispersion curve (Fig. 1). Creating a Love wave device with a particular guiding layer thickness places the operating point of the device at a specific point on the dispersion curve. The fractional change in frequency that occurs on deposition of mass onto the guiding layer surface then depends on the slope of the dispersion curve at that operating point. Upon increasing guiding layer thickness, the step-like changes in the plate mode frequencies show how large the change in slope can be with a guiding layer, and are therefore direct experimental evidence of enhanced mass sensitivity. As a simple estimate, the first transition of the highest-order plate mode, from $z=0.17$ to $z=0.36$, has a frequency change $\sim 21 \mathrm{MHz}$. In comparison, the frequency change of the first Love wave mode, estimated from $z=0.15$ to $z=0.22$, is $\sim 46 \mathrm{MHz}$. The effect of the guiding layer is to improve the plate mode sensitivity so that it comes within an order of magnitude of the Love wave. Figure 1 provides confirmation that guided plate modes exist, that higher-order Love waves on a finite thickness substrate are continuations from APMs, and that a guiding layer significantly enhances the mass sensitivity of the plate modes.

In a plate mode device, both the upper and lower faces PROof COPAderofs significant displacement so that either can be used to

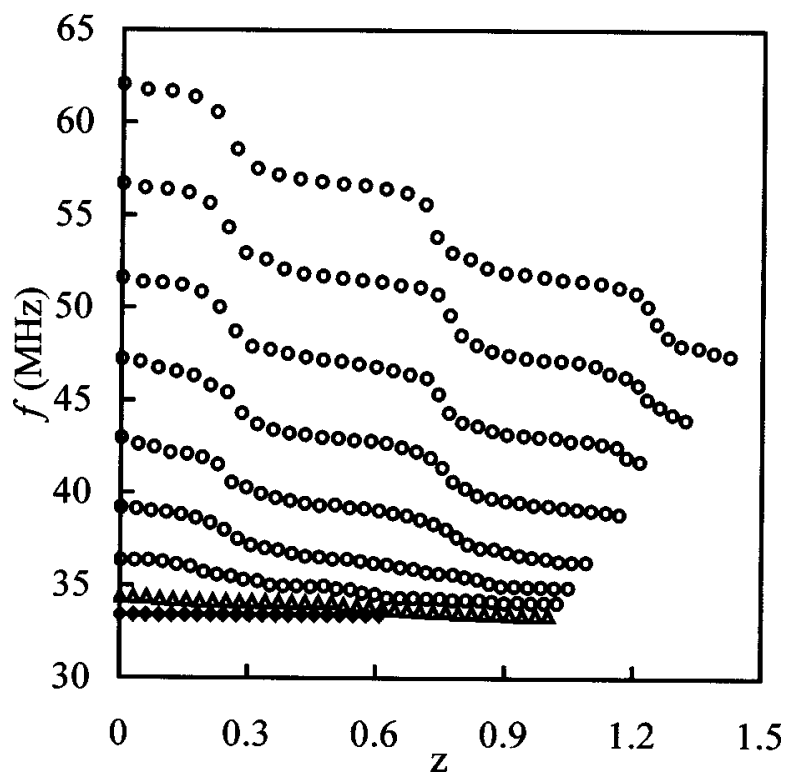

FIG. 2. Frequency of minimum insertion loss of the SSBW (solid diamonds), the lowest-order plate mode (open triangles) and the higher-order plate modes (open circles) and as a function of the normalized thickness $z$ of polymer coating on the face opposite to the transducers.

generate and detect the modes and either can be used for sensing. This contrasts with a Love wave device, in which only the face with the IDTs has significant displacement and must also therefore be used for sensing. Figure 2 shows the experimental data produced by coating the wave-guiding layer onto the face opposite to the transducers. The plate modes have $f>f_{\mathrm{SSBW}}$ and are indicated by open triangles and circles, while the SSBW mode is indicated by solid diamonds. In a similar way to Fig. 1, the plate modes are layer guided, and successively cascade in frequency towards lower order modes. A significant difference in Fig. 2 is that the SSBW mode does not change significantly in frequency and is no longer converted into a Love wave. At $z \sim 0.55$, when the frequency of the lowest plate mode (open triangles) reaches the frequency $f_{\mathrm{SSBW}}$ of the (unloaded) SSBW (i.e., where the second Love mode starts in Fig. 1), the SSBW mode vanishes into noise level. The step-like changes in the plate modes in Fig. 2 confirms that the mass sensitivity of the plate modes is enhanced by the guiding layer, even though, in this experiment, the transducers are located on the opposing face of the substrate. Figure 2 shows that layer-guided APMs do exist even when the transducers are located on the opposing face of the substrate, and that the enhancement in mass sensitivity by the use of a guiding layer can again be achieved.

In conclusion, Love wave and layer-guided acoustic plate modes have been generated using the same guiding layer and substrate. The data show that the traditional plate modes can be wave-guided in a similar way to Love waves and that the higher-order Love modes can be regarded as continuations of the layer-guided plate modes. As a result, the layer-guided plate modes exhibit an enhanced mass sensitivity compared to the traditional plate mode, while retaining all the advantages of operating via liquid sensing on the opposite face to the transducers. 
${ }^{1}$ E. Gizeli, A. C. Stevenson, N. J. Goddard, and C. R. Lowe, IEEE Trans. Ultrason. Ferroelectr. Freq. Control 39, 657 (1992).

${ }^{2}$ A. J. Ricco and S. J. Martin, Appl. Phys. Lett. 50, 1474 (1987).

${ }^{3}$ S. J. Martin, A. J. Ricco, T. M. Niemczyk, and G. C. Frye, Sens. Actuators 20, 253 (1989).
${ }^{4}$ G. McHale, M. I. Newton, and F. Martin, J. Appl. Phys. 91, 9701 (2002).

${ }^{5}$ M. I. Newton, G. McHale, and F. Martin, J. Appl. Phys. 91, 5735 (2002).

${ }^{6}$ E. Gizeli, Anal. Chem. 72, 5967 (2000).

${ }^{7}$ G. McHale, M. I. Newton, and F. Martin, J. Appl. Phys. (in press). 